\title{
REFLECTION FUNCTORS FOR HEREDITARY ALGEBRAS
}

\section{CLAUS MICHAEL RINGEL}

In this paper, we are mainly interested in representations of the quiver $\Gamma_{a b}$

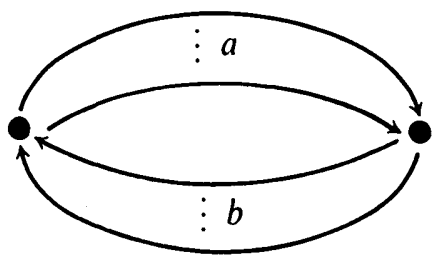

with $a$ arrows going from left to right, and $b$ arrows going from right to left, where $1 \leqslant b \leqslant a$. Thus, we consider the problem of normal forms of $a+b$-tuples of $n \times m$ matrices $A_{1}, \ldots, A_{a}$, and $m \times n$-matrices $B_{1}, \ldots, B_{b}$, with entries in some fixed commutative field $k$, with respect to the operation

$$
P A_{i} Q^{-1} \quad(1 \leqslant i \leqslant a), \quad Q B_{j} P^{-1} \quad(1 \leqslant j \leqslant b),
$$

where $P, Q$ are regular matrices of size $n \times n$ and $m \times m$, respectively. We call the pair $(n, m)$ the dimension type of this $a+b$-tuple, and we are concerned with the determination of the dimension types of the indecomposable representations. For the case where $b=0$, this question has been answered in [3], where it was shown that the dimension types of the indecomposable representations are precisely the positive roots of the corresponding root system. We will show that the same is true also when $b \geqslant 1$. However, whereas in the case when $b=0$, for every positive root there exists an indecomposable representation with trivial endomorphism ring, this is no longer true in general. Note that for $b \geqslant 1$, we will show that there are always even imaginary roots without indecomposable representations with trivial endomorphism ring, in contrast to a recent conjecture by $\mathrm{Kac}$ [2].

To be more precise, recall the definition of the root system in our case. Consider on $\mathbb{R}^{2}$ the quadratic form $q(x, y)=x^{2}+y^{2}-(a+b) x y$. The integral vectors $(x, y)$ with $q(x, y) \leqslant 0$ are called imaginary roots; the integral vectors $(x, y)$ with $q(x, y)=1$ are called real roots.

THEOREM. For every positive real root, there exists precisely one indecomposable representation of $\Gamma_{a b}$; for every positive imaginary root $(n, m)$, there exists a family of indecomposable representations of $\Gamma_{a b}$ with $-q(n, m)+1$ parameters. There are indecomposable representations with endomorphism ring a division ring only for the roots $(1,0),(0,1)$, and the imaginary roots $(n, m)$ with $\frac{1}{a} n \leqslant m \leqslant a n$.

Received 23 April, 1979.

[J. LoNDON MATh. Soc. (2), 21 (1980), 465-479] 
The main technique of the present paper will be a new type of reflection functor which realizes for representations the action of the generating reflections of the corresponding Weyl group. This functor will be constructed in the more general situation of a hereditary algebra and a given module $S$ with $\operatorname{End}(S)$ a division ring and Ext $(S, S)=0$. The $S$-reflection $\sigma_{S}(X)$ of a module $X$ (if it exists) will have the dimension type

$$
\operatorname{dim} \sigma_{s}(X)=\operatorname{dim} X-\frac{2(\operatorname{dim} X, \operatorname{dim} S)}{(\operatorname{dim} S, \operatorname{dim} S)} \operatorname{dim} S,
$$

as it should be. However, we stress the fact that we are able to define the $S$-reflection only for a very narrow class of modules. In contrast to the reflection functors constructed by Bernstein-Gelfand-Ponomarev [1], the image $\sigma_{s}(X)$ is a module over the same ring as the module $X$.

\section{Adding and removing bricks}

In this section, we are working with left modules of finite length over some fixed hereditary algebra. We will call them just modules, and we denote the corresponding category by $\mathfrak{M}$. Maps will be written on the right: thus for $f: X \rightarrow Y, g: Y \rightarrow Z$, the composition will be denoted by $f g$. We fix throughout this section some module $S$ with End $(S)$ a division ring and $\operatorname{Ext}(S, S)=0$.

If $\mathfrak{C}$ is a full subcategory of $\mathfrak{M}$, let $\mathfrak{C} / S$ be the quotient category of $\mathfrak{C}$ modulo the maps which factor through direct sums of copies of $S$. Note that the indecomposable objects of $\mathfrak{C} / S$ correspond precisely to the indecomposable modules in $\mathfrak{C}$ which are not isomorphic to $S$.

We consider the following four full subcategories of $\mathfrak{C}$. Let $\mathfrak{M}^{S}$ be given by the set of the modules $X$ with $\operatorname{Ext}(S, X)=0$ such that, in addition, $X$ has no direct summand which can be embedded into some direct sum of copies of $S$. Similarly, let $\mathfrak{M}_{S}$ be given by the set of modules $X$ with $\operatorname{Ext}(X, S)=0$ such that, in addition, no direct summand of $X$ is a quotient of a direct sum of copies of $S$. Finally, let $\mathfrak{M}^{-S}$ be given by the set of modules $X$ with $\operatorname{Hom}(X, S)=0$, and $\mathfrak{M}_{-s}$ by the set of modules $X$ with $\operatorname{Hom}(S, X)=0$. Also, we will consider intersections of these subcategories, namely

$$
\begin{aligned}
\mathfrak{M}_{s}^{S} & =\mathfrak{M}^{s} \cap \mathfrak{M}_{s}, \quad \mathfrak{M}_{-s}^{s}=\mathfrak{M}^{s} \cap \mathfrak{M}_{-s} \\
\mathfrak{M}_{s}^{-s} & =\mathfrak{M}^{-s} \cap \mathfrak{M}_{s}, \quad \mathfrak{M}_{-s}^{-s}=\mathfrak{M}^{-s} \cap \mathfrak{M}_{-s} .
\end{aligned}
$$

Given a module $X$, denote by $X^{-s}$ the intersection of the kernels of the maps $X \rightarrow S$. This induces a functor, since for $f: X \rightarrow Y$, we easily see that $\left(X^{-S}\right) f \subseteq Y^{-s}$.

Lemma 1. For any $X$, we have that $X^{-s}$ belongs to $\mathfrak{M}^{-s}$.

Proof. Let $\varphi: X^{-S} \rightarrow S$ be any map. Denote the exact sequence

$$
0 \rightarrow X^{-S} \hookrightarrow X \rightarrow V \rightarrow 0
$$


with $V=X / X^{-S}$ by $E$; thus $E \varphi^{*}$ belongs to $\operatorname{Ext}(V, S)$. However, $\operatorname{Ext}(V, S)=0$, since on the one hand, there is an embedding $\mu: V \rightarrow \bigoplus_{v} S$ for some $v \in \mathbb{N}$, so that

$$
\operatorname{Ext}(\mu, S): \operatorname{Ext}\left(\bigoplus_{v} S, S\right) \rightarrow \operatorname{Ext}(V, S)
$$

is surjective, and, on the other hand,

$$
\operatorname{Ext}\left(\bigoplus_{v} S, S\right) \approx \bigoplus_{v} \operatorname{Ext}(S, S)=0
$$

This shows that $\varphi$ can be extended to $X$; but the kernel of the extended map has to contain $X^{-s}$, and thus $\varphi=0$.

Lemma 2. Let $X \in \mathfrak{M}^{S}$, and $\varphi_{1}, \ldots, \varphi_{v}$ be a basis of $\operatorname{Hom}(X, S)_{\mathrm{End}(S)}$. Then the sequence

$$
0 \longrightarrow X X^{-S} \subset X \stackrel{\left(\varphi_{1}, \ldots, \varphi_{v}\right)}{\longrightarrow} \bigoplus_{v} S \longrightarrow 0
$$

is exact, and the induced sequences $E_{1}, \ldots, E_{v}$ form a basis of $f_{\operatorname{End}(S)} \operatorname{Ext}\left(S, X^{-S}\right)$.

Proof. Clearly, we may suppose that $X$ is indecomposable. Let $\varphi=\left(\varphi_{1}, \ldots, \varphi_{v}\right): X \rightarrow \bigoplus S$. Clearly, $X^{-S}$ is the kernel of $\varphi$. For, if $\varphi^{\prime}: X \rightarrow S$, then $\varphi^{\prime}=\sum_{i=1}^{v} \varphi_{i} \alpha_{i}$ for some $\alpha_{i} \in \operatorname{End}(S)$, thus $\varphi^{\prime}=\varphi \alpha$ with $\alpha=\left(\begin{array}{c}\alpha_{1} \\ \vdots \\ \alpha_{v}\end{array}\right): \bigoplus_{v} S \rightarrow S$, and $\operatorname{Ker} \varphi \subseteq \operatorname{Ker} \varphi^{\prime}$.

Let $V$ be the image of $\varphi$, with inclusion map $\mu: V \rightarrow \bigoplus_{v} S$. Since $\operatorname{Ext}\left(\mu, X^{-S}\right)$ is surjective, there exists an exact sequence $E \in \operatorname{Ext}\left(\bigoplus_{v} S, X^{-s}\right)$ such that $\mu^{*} E$ is the given sequence

$$
0 \rightarrow X^{-s} \hookrightarrow X \rightarrow V \rightarrow 0 .
$$

Let $E_{i} \in \operatorname{Ext}\left(S, X^{-S}\right)$ be the induced sequences of $E$ with respect to the canonical inclusions $S \rightarrow \bigoplus_{v} S$. Since

$$
\operatorname{Hom}(S, V) \rightarrow \operatorname{Ext}\left(S, X^{-S}\right) \rightarrow \operatorname{Ext}(S, X)
$$

is exact, and by assumption $\operatorname{Ext}(S, X)=0$, we see that $E_{i}=\psi_{i}^{*} \mu^{*} E$ for some map $\psi_{i}: S \rightarrow V$. Let $\psi=\left(\begin{array}{c}\psi_{1} \\ \vdots \\ \psi_{v}\end{array}\right): \bigoplus_{v} S \rightarrow V$; then $E=\psi^{*} \mu^{*} E$, and therefore $\mu^{*} E=$ 
$\mu^{*} \psi^{*} \mu^{*} E$. Thus, we have a commutative diagram

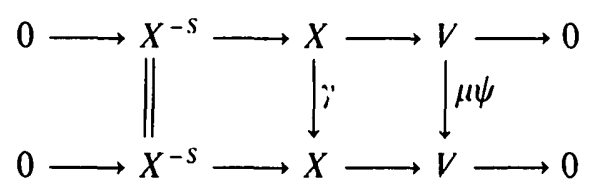

with some $\gamma \in$ End $(X)$. Since $X$ is indecomposable, $\gamma$ is either nilpotent or an automorphism. But since $X \in \mathfrak{P}^{s}$, we know that $X^{-s} \neq 0$, thus $\gamma$ cannot be nilpotent. This shows that $\gamma$ and therefore also $\mu \psi$ is an automorphism. Consequently, $\mu$ is a split monomorphism, and its cokernel is a direct sum of copies of $S$. Let $\pi=\left(\begin{array}{c}\pi_{1} \\ \vdots \\ \pi_{v}\end{array}\right): \oplus S \rightarrow S$ be any map with $\mu \pi=0$. Then $0=\varphi \pi=\sum \varphi_{i} \pi_{i}$ implies that all $\pi_{i}=0$, since $\varphi_{1}, \ldots, \varphi_{v}$ is a basis of $\operatorname{Hom}(X, S)_{\text {End(SS. }}$. This shows that the cokernel of $\mu$ is zero, thus $\mu$ is an isomorphism.

Note that any $\beta: S \rightarrow X$ maps into $X^{-S}$, since otherwise $X$ would split off a copy of $S$. Consequently, the canonical map $\operatorname{Hom}\left(S, X^{-S}\right) \rightarrow \operatorname{Hom}(S, X)$ is an isomorphism, thus the long exact sequence for $\operatorname{Hom}(S,-)$ gives rise to an isomorphism

$$
\operatorname{Hom}\left(S, \bigoplus_{v} S\right) \rightarrow \operatorname{Ext}\left(S, X^{-S}\right)
$$

using also that fact that $\operatorname{Ext}(S, X)=0$. This shows that the exact sequences $E_{1}, \ldots, E_{v}$ (which are the images of the canonical inclusion maps $S \rightarrow \bigoplus_{v} S$ ) form a basis of End $(S) \operatorname{Ext}\left(S, X^{-S}\right)$.

Lemma 3. Let $Y \in \mathfrak{M}^{-S}$ and let $E_{1}, \ldots, E_{v}$ be a basis of $\operatorname{End}(S) \operatorname{Ext}(S, Y)$. Consider the exact sequence $E$

$$
0 \rightarrow Y \rightarrow Z \rightarrow \bigoplus_{\nu} S \rightarrow 0
$$

given by the elements $E_{i}$. Then $Z \in \mathfrak{M}^{S}$, and $Z^{-S} \approx Y$.

Proof. Applying $\operatorname{Hom}(S,-)$ to $E$, we obtain the exact sequence

$$
\operatorname{Hom}\left(S, \bigoplus_{v} S\right) \rightarrow \operatorname{Ext}(S, Y) \rightarrow \operatorname{Ext}(S, Z) \rightarrow \operatorname{Ext}\left(S, \bigoplus_{v} S\right)
$$

By construction, the first map is an isomorphism, and since in addition $\operatorname{Ext}(S, \underset{v}{\oplus} S)=0$, we conclude that $\operatorname{Ext}(\dot{S}, Z)=0$. Also, assume that $Z=Z_{1} \oplus Z_{2}$ with an embedding $Z_{2} \hookrightarrow \oplus S$. Since $\operatorname{Hom}(Y, S)=0$, the image of $Y$ under the given map $\imath: Y \rightarrow Z$ has to be contained in $Z_{1}$. Thus the cokernel of $\imath$ is isomorphic to the direct sum of the cokernel of the inclusion $Y \rightarrow Z_{1}$ and the module $Z_{2}$. In particular, $Z_{2}$ is a direct sum of copies of $S$. Assume that $Z_{2} \neq 0$; then any direct summand of the form $S$ of $Z_{2}$ gives rise to an inclusion $\alpha=\left(\alpha_{1}, \ldots, \alpha_{v}\right): S \rightarrow \bigoplus_{v} S$ such that $\alpha^{*} E=0$. 
However, this means that $\sum_{i=1}^{v} \alpha_{i} E_{i}=0$ which contradicts the fact that the elements $E_{1}, \ldots, E_{v}$ are linearly independent. Thus, we have shown that $Z \in \mathfrak{M}^{S}$. It is clear that $Z^{-S}=\imath(Y)$, since $\operatorname{Hom}(Y, S)=0$.

Proposimion 1. The functor $X \mapsto X^{-S}$ gives an equivalence of the category $\mathfrak{M}^{S} / S$ onto the category $\mathfrak{M}^{-S}$.

Proof. Let us first show that this functor $\mathfrak{M}^{S} \rightarrow \mathfrak{M}^{-s}$ is full. Let $X, Y \in \mathfrak{M}^{S}$ with the canonical sequences

$$
\begin{aligned}
& E: 0 \longrightarrow X^{-s} \subset X \stackrel{\left(\varphi_{1}, \ldots, \varphi_{v}\right)}{\longrightarrow} \bigoplus_{v} S \longrightarrow 0, \\
& F: 0 \longrightarrow Y^{-s} \longrightarrow Y \stackrel{\left(\psi_{1}, \ldots, \psi_{w}\right)}{\longrightarrow} \underset{w}{\oplus} S \longrightarrow 0,
\end{aligned}
$$

of Lemma 2.

Given $g: X^{-s} \rightarrow Y^{-s}$, we form the induced sequence $E^{\prime}=E g^{*}$

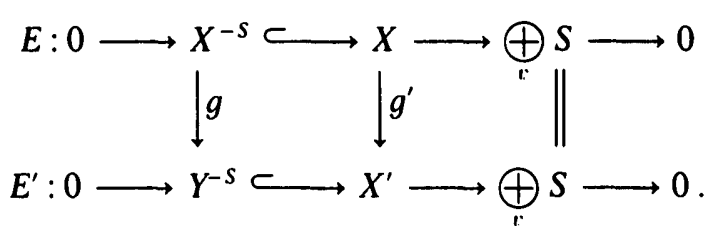

Now $E^{\prime}$ gives rise to $v$ exact sequences $E_{i}^{\prime} \in \operatorname{Ext}\left(S, Y^{-S}\right)$, and we can write them as linear combinations with respect to the basis $F_{1}, \ldots, F_{w}$ of ${ }_{\operatorname{End}(S)} \operatorname{Ext}\left(S, Y^{-S}\right)$ as follows: $E_{i}^{\prime}=\sum_{j=1}^{w} \alpha_{i j} F_{j}$. In this way, we obtain a commutative diagram

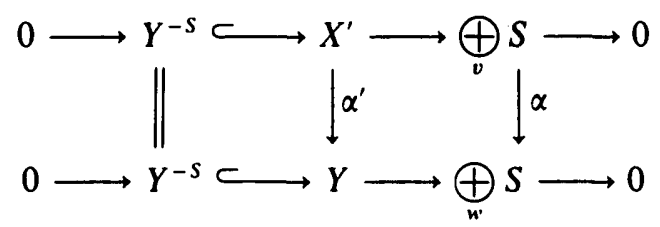

with $\alpha=\left(\alpha_{i j}\right)$. As a consequence $g: X^{-S} \rightarrow Y^{-S}$ is the restriction of the map $g^{\prime} \alpha^{\prime}: X \rightarrow Y$.

On the other hand, if $f: X \rightarrow Y$ induces the zero map $f^{-s}: X^{-s} \rightarrow Y^{-s}$, then the restriction of $f$ to $X^{-s}$ is zero, thus $f$ factors over $X / X^{-s}=\bigoplus_{v} S$. Conversely, assume that $f: X \rightarrow Y$ factors over some direct sum $\oplus S$ of copies of $S$. Then it is clear that $f^{-s}: X^{-s} \rightarrow Y^{-s}$ is zero.

Since by Lemma 3 , any module in $\mathfrak{M}^{-s}$ is of the form $Z^{-s}$ for some $Z \in \mathfrak{M}^{s}$, we see that the functor $X \mapsto X^{-s}$ gives an equivalence between $\mathfrak{M}^{s} / S$ and $\mathfrak{M}^{-s}$. 
Let us formulate the corresponding dual assertion:

Proposition $1^{*}$. The categories $\mathfrak{M}_{S} / S$ and $\mathfrak{M}_{-s}$ are equivalent under the functor $X \mapsto X_{-s}=X / X^{\prime}$, where $X^{\prime}$ is the sum of the images of all maps $S \rightarrow X$.

Let $K(\mathfrak{M})$ be the Grothendieck group of $\mathfrak{M}$ with respect to all short exact sequences. Thus $K(\mathfrak{M})$ can be identified with the free abelian group generated by the isomorphism classes of simple modules. The canonical map from $\mathfrak{M N}$ into $K(\mathfrak{M N})$ will be denoted by $\operatorname{dim}$. Let $k$ be the commutative field over which $\mathfrak{M}$ is defined. There is a (usually nonsymmetric) bilinear form on $K(\mathfrak{M})$ given by

$$
\langle\operatorname{dim} X, \operatorname{dim} Y\rangle=\operatorname{dim}_{k} \operatorname{Hom}(X, Y)-\operatorname{dim}_{k} \operatorname{Ext}(X, Y),
$$

due to the fact that $\mathfrak{M}$ is hereditary. Usually, we will denote $\langle\operatorname{dim} X, \operatorname{dim} Y\rangle$ just by $\langle X, Y\rangle$. The corresponding symmetric bilinear form (the symmetrization of $\langle$, $\rangle$ ) will be denoted by $($,$) . Note that any element s \in K(\mathfrak{M})$ with $(s, s) \neq 0$ gives rise to a reflection $\sigma_{s}$ of $K(\mathfrak{M})$ defined by

$$
\sigma_{s}(x)=x-\frac{2(x, s)}{(s, s)} s .
$$

Proposition 2. There is an equivalence of categories $\sigma_{s}: \mathfrak{M}_{S}^{S} / S \rightarrow \mathfrak{M}_{-S}^{-S}$, and an equivalence of categories $\sigma_{s}: \mathfrak{M}_{s}^{-s} \rightarrow \mathfrak{M}_{-s}^{s}$ such that

$$
\operatorname{dim} \sigma_{S}(X)=\sigma_{\operatorname{dim} S}(\operatorname{dim} X) .
$$

Remark. These equivalences, and also their inverses, all are denoted by $\sigma_{s}$, since it will be seen that they coincide on the mutual intersections of the categories $\mathfrak{M}_{s}^{s}, \mathfrak{M}_{s}^{-s}, \mathfrak{M}_{-s}^{s}$ and $\mathfrak{M}_{-s}^{-s}$

Proof. For $X \in \mathfrak{M}_{S}^{S}$, define $\sigma_{S}(X)=X^{-S} / X^{\prime}$, where $X^{\prime}$ is the sum of the images of all maps $S \rightarrow X$. Note that since $X$ does not split off a copy of $S$, we have $X^{\prime} \subseteq X^{-s}$. It follows from the Propositions 1 and $1^{*}$ that $\sigma_{s}$ induces an equivalence from $\mathfrak{M}_{S}^{S} / S$ onto $\mathfrak{M}_{-s}^{-S}$. Now $X^{-S}$ is obtained from $X$ by removing $\operatorname{dim} \operatorname{Hom}(X, S)_{\mathrm{End}(S)}$ copies of $S$, and $X^{\prime}$ is the direct sum of $\operatorname{dim}_{\text {End(S) }} \operatorname{Hom}(S, X)$ copies of $S$, thus

$$
\begin{aligned}
\operatorname{dim} \sigma_{S}(X) & =\operatorname{dim} X-\left(\operatorname{dim} \operatorname{Hom}(X, S)_{\mathrm{End}(S)}+\operatorname{dim}_{\mathrm{End}(S)} \operatorname{Hom}(S, X)\right) \operatorname{dim} S \\
& =\operatorname{dim} X-\frac{\langle X, S\rangle+\langle S, X\rangle}{\langle S, S\rangle} \operatorname{dim} S \\
& =\sigma_{\operatorname{dim} S}(\operatorname{dim} X)
\end{aligned}
$$

using the facts that $\operatorname{Ext}(X, S)=\operatorname{Ext}(S, X)=\operatorname{Ext}(S, S)=0$, that

$$
\operatorname{dim}_{k} \operatorname{Hom}(X, S)=\operatorname{dim}_{k} \operatorname{End}(S) \cdot \operatorname{dim} \operatorname{Hom}(X, S)_{\text {End }(S)},
$$

and similarly for $\operatorname{dim}_{k} \operatorname{Hom}(S, X)$. 
Next, let $X \in \mathfrak{M}_{S}^{-S}$. Let $E_{1}, \ldots, E_{v}$ be a basis of ${ }_{\text {End (S) }} \operatorname{Ext}(S, X)$, and let

$$
0 \rightarrow X \hookrightarrow Z \rightarrow \bigoplus_{v} S \rightarrow 0
$$

be the exact sequence given by the elements $E_{i}$. Let $X^{\prime}$ be the sum of the images of the maps $S \rightarrow X$. Define $\sigma_{S}(X)=Z / X^{\prime}$. Note that in both categories $\mathcal{M}_{S}^{-S}$ and $\mathcal{M U}_{-s}^{S}$ there are no non-zero maps which factor through direct sums of copies of $S$. Thus, the Propositions 1 and $1^{*}$ show that $\sigma_{s}$ is an equivalence from $\mathrm{JJl}_{S}^{-s}$ onto $\mathrm{Jjl}_{-s}^{S}$. Also,

$$
\begin{aligned}
\operatorname{dim} \sigma_{S}(X) & =\operatorname{dim} X+\operatorname{dim} \operatorname{Ext}(X, S)_{\mathrm{End}(S)} \cdot \operatorname{dim} S-\operatorname{dim}_{\mathrm{End}(S)} \operatorname{Hom}(S, X) \cdot \operatorname{dim} S \\
& =\operatorname{dim} X-\frac{\langle X, S\rangle+\langle S, X\rangle}{\langle S, S\rangle} \operatorname{dim} S \\
& =\sigma_{\operatorname{dim} S}(\operatorname{dim} X)
\end{aligned}
$$

The equivalence of categories $\sigma_{s}: \mathfrak{M}_{s}^{-S} \rightarrow \mathfrak{M}_{-S}^{s}$ means, in particular, that we may identify for $X \in \mathfrak{M}_{S}^{-s}$ the rings End $(X)$ and End $\sigma_{S}(X)$. For $X \in \mathfrak{M}_{S}^{S}$, the endomorphism rings of $X$ and $\sigma_{S}(X)$ will usually differ, and we note the following formula for the change of dimension:

Proposition 3. Let $X \in \mathfrak{M}_{s}^{S}$. Then

$$
\operatorname{dim}_{k} \operatorname{End} \sigma_{S}(X)=\operatorname{dim}_{k} \operatorname{End}(X)-\frac{\langle X, S\rangle \cdot\langle S, X\rangle}{\langle S, S\rangle} .
$$

Proof. By Proposition 2, there is a surjective ring homomorphism End $(X) \rightarrow$ End $\sigma_{s}(X)$, with kernel

$$
\{\varphi \in \operatorname{End}(X) \mid \varphi \text { factorises over some } \oplus S\} .
$$

As a $k$-vector space, this ideal is isomorphic to

$$
\operatorname{Hom}\left(X / X^{-s}, X^{\prime}\right) \approx \operatorname{Hom}\left(\bigoplus_{v} S, \underset{u}{\oplus} S\right) \approx \bigoplus_{u v} \operatorname{Hom}(\dot{S}, S)
$$

where, as before, $X^{\prime}$ is the sum of the images of maps $S \rightarrow X$, and

$$
\begin{aligned}
& v=\operatorname{dim} \operatorname{Hom}(X, S)_{\mathrm{End}(S)}=\frac{\langle X, S\rangle}{\langle S, S\rangle}, \\
& u=\operatorname{dim}_{\mathrm{End}(S)} \operatorname{Hom}(S, X)=\frac{\langle S, X\rangle}{\langle S, S\rangle} .
\end{aligned}
$$

Thus, the $k$-dimension of this ideal is

$$
u \cdot v \cdot \operatorname{dim}_{k} \operatorname{End}(S)=\frac{\langle X, S\rangle \cdot\langle S, X\rangle}{\langle S, S\rangle}
$$


Proposition 3*. Let $X \in \mathfrak{M}_{-S}^{-S}$. Then

$$
\operatorname{dim}_{k} \operatorname{End} \sigma_{S}(X)=\operatorname{dim}_{k} \operatorname{End}(X)+\frac{\langle X, S\rangle\langle S, X\rangle}{\langle S, S\rangle}
$$

Usually it will be of interest, to repeat this construction several times, using different modules $S$. Rather weak conditions suffice in order to be able to do so. For example, we will use in the next section the following lemma.

Lemma 4. Let $S, T$ be modules, where $T$ is simple.

(i) If $\operatorname{Ext}(S, T) \neq 0$, then $\mathfrak{M}^{S} \subseteq \mathfrak{M}^{-T}$.

(ii) If $\operatorname{Ext}(T, S) \neq 0$, then $\mathfrak{M}_{S} \subseteq \mathfrak{M}_{-T}$.

Proof. Let $X \in \mathfrak{M}^{S}$, and assume there exists a non-zero map $\varphi: X \rightarrow T$. Since $T$ is simple, $\varphi$ has to be surjective, thus also $\operatorname{Ext}(S, \varphi): \operatorname{Ext}(S, X) \rightarrow \operatorname{Ext}(S, T)$ is surjective. Since $\operatorname{Ext}(S, T) \neq 0$, this contradicts the fact that $\operatorname{Ext}(S, X)=0$. Similarly, if $Y \in \mathfrak{D}_{S}$, and $\varphi: T \rightarrow Y$ is a non-zero map, then it is a monomorphism, thus $\operatorname{Ext}(\varphi, S): \operatorname{Ext}(Y, S) \rightarrow \operatorname{Ext}(T, S)$ is surjective.

\section{Construction of indecomposable representations of $\Gamma_{a b}$}

We consider now the special case of the representations of $\Gamma_{a b}$ with $a \geqslant b \geqslant 1$ and our aim is to prove the theorem formulated in the introduction. We work over a fixed field $k$.

We denote by $V=\left(V_{1}, \ldots, V_{a}, V_{1}^{\prime}, \ldots, V_{b}^{\prime}\right)$ a representation of $\Gamma_{a b}$, say of dimension type $(n, m)$ where the $V_{i}$ are $n \times m$-matrices, and the $V_{i}^{\prime}$ are $m \times n$-matrices. The set of all representations of dimension type $(n, m)$ will be denoted by $\mathscr{R}(n, m)$ : it is an affine space over $k$ of dimension $(a+b) n m$, and the isomorphism classes are just the orbits in $\Re(n, m)$ of the obvious action by the algebraic group $\mathrm{GL}_{n}(k) \times \mathrm{GL}_{m}(k)$. We denote by $S$ the unique representation of dimension type $(1,0)$, by $T$ the one of type $(0,1)$. The Grothendieck group of the representations of $\Gamma_{a b}$ with respect to short exact sequences canonically maps onto $\mathbb{Z}^{2}$, and the induced bilinear form $\langle$,$\rangle on \mathbb{Z}^{2}$ is given by

$$
\left\langle(x, y),\left(x^{\prime}, y^{\prime}\right)\right\rangle=x x^{\prime}+y y^{\prime}-a x y^{\prime}-b x^{\prime} y,
$$

see [3]. As before, the corresponding quadratic form is denoted by $q$, and we let $p(n, m)=-q(n, m)+1$.

Lemma 5. Let $(n, m) \in \mathbb{N}^{2}$, with $\frac{1}{b} n \leqslant m \leqslant b n$. Then there exists a $p(n, m)$ parameter family $\mathscr{U}(n, m)$ of isomorphism classes of representations $V$ with End $(V)=k$, and $\operatorname{Hom}(S, V)=\operatorname{Hom}(V, S)=\operatorname{Hom}(T, V)=\operatorname{Hom}(V, T)=0, \quad$ and with $\operatorname{dim} V=(n, m)$. 
Proof. Note that $\operatorname{Hom}(S, V)=0$ if and only if the $n \times$ am matrix $\left(V_{1}, V_{2}, \ldots, V_{n}\right)$ is left regular, that $\operatorname{Hom}(V, S)=0$ if and only if the $b m \times n$ matrix $\left(\begin{array}{c}V_{1}^{\prime} \\ \vdots \\ V_{b}^{\prime}\end{array}\right)$ is right regular, and similarly for $T$. Thus, the set $\mathscr{U}(n, m)$ of all representations $V$ with $\operatorname{End}(V)=k$ and

$$
\operatorname{Hom}(S, V)=\operatorname{Hom}(V, S)=\operatorname{Hom}(T, V)=\operatorname{Hom}(V, T)=0
$$

is open in $\mathscr{R}(n, m)$. If we show that $\mathscr{U}(n, m)$ is non-empty, then it is a variety of dimension $(a+b) n m$ consisting of orbits of equal dimension, thus there remain $p(n, m)$ parameters.

Consider first the dimension types $(1, c)$ with $1 \leqslant c \leqslant b$. We construct one particular representation $V=V(c) \in \mathscr{R}(1, c)$ as follows: For $1 \leqslant i \leqslant c$, let $V_{i}$ be the $1 \times c$ elementary matrix $(0 \ldots 010 \ldots 0)$ with 1 in the $i$-th position, and $V_{i}^{\prime}$ the transpose of $V_{i}$, whereas for $i>c$, let $V_{i}=0, V_{i}^{\prime}=0$. It is easy to see that $V$ belongs even to $\mathscr{U}(1, c)$ and, moreover, for $1 \leqslant c, d \leqslant b$, we have $\operatorname{Hom}(V(c), V(d))=0$.

Next, let $n \leqslant m \leqslant b n$. There are natural numbers $u, v$ with

$$
(n, m)=u(1, c)+v(1, c+1)
$$

for some $1 \leqslant c<b$. In order to construct a representation in $\mathscr{U}(n, m)$, we use the method of simplification as presented in [3]. Namely, $V(c)$ and $V(c+1)$ are orthogonal points in the terminology of that paper. Note that

$$
\operatorname{Ext}(V(c), V(c)) \neq 0, \quad \operatorname{Ext}(V(c+1), V(c+1)) \neq 0,
$$

and also

$$
\operatorname{Ext}(V(c+1), V(c)) \neq 0
$$

since

$$
\langle(1, c+1),(1, c)\rangle<0
$$

thus there exists a representation $W$ of type $(n, m)$ with a chain of subrepresentations

$$
0=W^{0} \subset W^{1} \subset \ldots \subset W^{u+v}=W,
$$

where the lower $u$ factors $W^{i} / W^{i-1}(1 \leqslant i \leqslant u)$ are isomorphic to $V(c)$, and the upper $v$ factors $W^{i} / W^{i-1}(u+1 \leqslant i \leqslant u+v)$ are isomorphic to $V(c+1)$, such that, moreover, the factors $W^{i+1} / W^{i-1}(1 \leqslant i \leqslant u+v-1)$ are indecomposable. It follows that $W$ belongs to $\mathscr{U}(n, m)$.

For the dimension types $(n, m)$ with $\frac{1}{b} n \leqslant m \leqslant n$, we proceed similarly: First, we construct representations of type $(c, 1)$ with $1 \leqslant c \leqslant b$, which belong to $\mathscr{U}(c, 1)$ and have no homomorphisms between them, and then we form corresponding extensions.

Lemma 6. Let $(n, m) \in \mathbb{N}^{2}$ with $\frac{1}{a} n \leqslant m<\frac{1}{b} n$. Then there exists a $p(n, m)$ parameter family $\mathscr{U}(n, m)$ of isomorphism classes of representations $V$ with 
$\operatorname{End}(V)=k, \quad$ and $\quad \operatorname{Hom}(S, V)=\operatorname{Hom}(T, V)=\operatorname{Hom}(V, T)=0 \quad$ and $\quad$ with $\operatorname{dim} V=(n, m)$.

Proof. Again, we only have to construct one particular representation with these properties. Let $n=b m+s$ with $0 \leqslant s \leqslant(a-b) m$. Now use the previous lemma for the dimension type $(b m, m)$. In this way, we obtain a representation $W$ of type $(b m, m)$ such that $\operatorname{End}(W)=k$, and

$$
\operatorname{Hom}(S, W)=\operatorname{Hom}(W, S)=\operatorname{Hom}(T, W)=\operatorname{Hom}(W, T)=0 .
$$

We can simplify $W$ and $S$ at the same time, since they are orthogonal points. Now, since $\operatorname{Hom}(S, W)=0$,

$$
\begin{aligned}
\operatorname{dim} \operatorname{Ext}(S, W) & =-\langle(1,0),(b m, m)\rangle \\
& =(a-b) m
\end{aligned}
$$

The representations $V$ of $\Gamma_{a b}$ with an exact sequence of the form

$$
0 \rightarrow \bigoplus_{u} W \rightarrow V \rightarrow \bigoplus_{v} S \rightarrow 0
$$

for some $u, v \in \mathbb{N}$ form a full subcategory which is equivalent to the category of representations of $\Gamma_{(a-b) m, 0}($ see [3]), and $(s, 1)$ is the dimension type of a representation of $\Gamma_{(a-b) m, 0}$ with endomorphism ring $k$, if and only if

$$
s^{2}+1^{2}-(a-b) m s \leqslant 1
$$

again according to [3]. In our case, this is satisfied according to $0 \leqslant s \leqslant(a-b) m$. Thus there exists $V$ with End $(V)=k$ and with an exact sequence

$$
0 \rightarrow W \rightarrow V \rightarrow \bigoplus_{s} S \rightarrow 0
$$

It is clear that $\operatorname{Hom}(S, V)=\operatorname{Hom}(T, V)=\operatorname{Hom}(V, T)=0$.

Similarly, we have the following lemma.

Lemma $6^{*}$. Let $(n, m) \in \mathbb{N}^{2}$ with $b n<m \leqslant$ an. Then there exists a $p(n, m)$ parameter family $\mathscr{U}(n, m)$ of isomorphism classes of representations $V$ with $\operatorname{End}(V)=k$ and $\operatorname{Hom}(S, V)=\operatorname{Hom}(V, S)=\operatorname{Hom}(V, T)=0$ and with $\operatorname{dim} V=(n, m)$.

For the proof, we consider representations $V$ with an exact sequence of the form

$$
0 \rightarrow \bigoplus_{t} T \rightarrow V \rightarrow W \rightarrow 0
$$

where $a n=b n+t$, and $W$ is a representation of dimension type $(n, b n)$ with the properties mentioned in Lemma 5. 
Proof of the theorem. Let $\sigma_{1}, \sigma_{2}$ be the reflections on $\mathbb{Z}^{2}$ defined by

$$
\sigma_{1}(x, y)=(-x+(a+b) y, y), \quad \sigma_{2}(x, y)=(x,-y+(a+b) x) ;
$$

note that they generate the Weyl group for our problem. It is easy to see that the set of positive imaginary roots is the disjoint union of

$$
\mathbf{B}=\left\{\begin{array}{l|l}
(n, m) \in \mathbb{N}^{2} & \frac{1}{a} n \leqslant m \leqslant a n
\end{array}\right\}
$$

and the various $\mathbf{S}_{i}, \mathbf{T}_{i}$, with $i \geqslant 1$, where

and

$$
\mathbf{S}_{i+1}=\sigma_{1}\left(\mathbf{T}_{i}\right), \quad \mathbf{T}_{i+1}=\sigma_{2}\left(\mathbf{S}_{i}\right),
$$

$$
\begin{aligned}
& \mathbf{S}_{0}=\left\{(n, m) \in \mathbb{N}^{2} \mid \frac{1}{a} n \leqslant m<b n\right\}, \\
& \mathbf{T}_{0}=\left\{(n, m) \in \mathbb{N}^{2} \mid \frac{1}{b} n<m \leqslant a n\right\} .
\end{aligned}
$$

For any imaginary root $(n, m)$ in $\mathbf{B}$ we have seen that there is a $p(n, m)$-parameter family $\mathscr{U}(n, m)$ of isomorphism classes of representations $V$ with $\operatorname{End}(V)=k$. In fact, in case $V \in \mathscr{U}(n, m)$ we know the following: if $(n, m) \in \mathbf{S}_{0}$, then $V \in \mathfrak{M}_{-T}^{-T}$, whereas if $(n, m) \in \mathbf{T}_{0}$, then $V \in \mathfrak{M}_{-s}^{-S}$. We now use induction on $i$, in order to construct for $(n, m) \in \mathbf{S}_{i}$, a $p(n, m)$-parameter family $\mathscr{U}(n, m)$ of representations $V$ with $V \in \mathfrak{M}_{-T}^{-T}$, and for $(n, m) \in \mathbf{T}_{i}$, a $p(n, m)$-parameter family $\mathscr{U}(n, m)$ of representations $V$ with $V \in \mathfrak{M}_{-s}^{-S}$. For, if $V \in \mathfrak{M}_{-s}^{-S}$, then we can use the $S$-reflection $\sigma_{S}$ in order to obtain a module $\sigma_{s}(V) \in \mathcal{M S S}_{S}^{S} \subseteq \mathfrak{M}_{-T}^{-T}$, whereas for $V \in \mathfrak{M}_{-T}^{-T}$, we use the $T$-reflection $\sigma_{T}$ in order to obtain a module $\sigma_{T}(V) \in \mathfrak{M}_{T}^{T} \subseteq \mathfrak{M}_{-S}^{-S}$.

Similarly, in case $(n, m)$ is a positive real root, then $(n, m)$ is obtained either from $(1,0)$ or $(0,1)$ by the application of some element $w$ of the Weyl group. Again, by induction on the length of $w$, we construct indecomposable representations

$$
\begin{array}{ll}
\sigma_{S} \sigma_{T} \ldots \sigma_{S} \sigma_{T}(S), & \sigma_{T} \sigma_{S} \sigma_{T} \ldots \sigma_{S} \sigma_{T}(S), \\
\sigma_{S} \sigma_{T} \sigma_{S} \ldots \sigma_{T} \sigma_{S}(T), & \sigma_{T} \sigma_{S} \ldots \sigma_{T} \sigma_{S}(T) .
\end{array}
$$

According to a theorem of $\mathrm{Kac}$ [2], there can be at most one indecomposable representation of dimension type a positive real root, thus the constructed representation is the only indecomposable one of dimension type the given positive real root.

It remains to be seen that for an indecomposable representation $V$ with $\operatorname{dim} V=(n, m)$ and $\operatorname{End}(V)$ a division ring, either $(n, m)=(1,0)$ or $(0,1)$, or else $\frac{1}{a} n \leqslant m \leqslant a n$. However, if $\frac{1}{a} n>m \geqslant 0$, then both

$$
\langle(1,0),(n, m)\rangle=n-a m>0,
$$


and

$$
\langle(n, m),(1,0)\rangle=n-b m \geqslant n-a m>0 ;
$$

thus $\operatorname{Hom}(S, V) \neq 0, \operatorname{Hom}(V, S) \neq 0$, and therefore there is an endomorphism of $V$ with image $S$. Similarly, one sees that for $m>a n \geqslant 0$, there will be an endomorphism of $V$ with image $T$.

This finishes the proof of the theorem.

The last part of the proof above implies that for some positive roots $(n, m)$, the set of indecomposable representations $V$ of dimension type $(n, m)$ will not be dense in $\mathscr{H}(n, m)$.

Let $e(n, m)$ be the smallest possible number which occurs as $\operatorname{dim} \operatorname{End}(W)$ with $W$ of dimension type $(n, m)$. The set of all representations $W$ of dimension type $(n, m)$ with $\operatorname{dim} \operatorname{End}(W)=e(n, m)$ is called the open sheet and will be denoted by $\mathscr{n}(n, m)^{0}$. We want to add that usually even for a positive imaginary root $(n, m)$, the open sheet does not contain any indecomposable representation.

Proposition 4. (a) If $(n, m)=(1,0)$ or $(0,1)$, or $\frac{1}{a} n \leqslant m \leqslant a n$, then $e(n, m)=1$; thus the open sheet consists of the (indecomposable) representations $V$ with $\operatorname{End}(V)=k$.

(b) If $(n, m)$ satisfies $0<m<\frac{1}{a} n$ then $e(n, m)=(n-a m)(n-b m)+1$; if $(n, m)$ satisfies $0<$ an $<m$, then $e(n, m)=(m-a n)(m-b n)+1$; and in both cases the open sheet contains decomposable representations.

(c) In case $(n, m)=(1,0)$, or $(0,1)$, or $(1, a+b)$, or $(a+b, 1)$, the indecomposable representations belong to the open sheet. In case $\frac{a}{a^{2}+a b-1} n \leqslant m \leqslant \frac{a^{2}+a b-1}{a} n$, the family $\mathscr{U}(n, m)$ constructed in this section belongs to the open sheet. For all other dimension types, the open sheet does not contain any indecomposable representation.

Proof. The assertion (a) has been shown above. For (b), let $0<m<\frac{1}{a} n$, and consider the representations of the form $V \oplus \bigoplus_{s} S$, where $n=a m+s$, and $V$ is a representation of dimension type $(a m, m)$ with $\operatorname{End}(V)=k$, and with an exact sequence of the form

$$
0 \rightarrow W \rightarrow V \rightarrow \bigoplus_{(a-b) m} S \rightarrow 0,
$$

where $W$ is of dimension type $(b m, m)$ and $\operatorname{Hom}(S, W)=0, \operatorname{Hom}(W, S)=0$. As we have seen in Lemma 6, there is a $p(a m, m)$-parameter family of such representations $V$. Note that $p(a m, m)=(a b-1) m^{2}-1$. We calculate the dimension of the endomorphism ring of $V \oplus \bigoplus_{s} S$ as follows:

$$
\begin{aligned}
\operatorname{dim} \operatorname{End}\left(V \oplus \bigoplus_{S} S\right) & =\operatorname{dim}_{k} \operatorname{End}(V)+s \cdot \operatorname{dim}_{k} \operatorname{Hom}(V, S)+s^{2} \operatorname{dim}_{k} \operatorname{End}(S) \\
& =1+s \cdot(a-b) m+s^{2}=(n-a m)(n-b m)+1 .
\end{aligned}
$$


Thus the orbit of any such representation has dimension

$$
\begin{aligned}
o(m, n) & =m^{2}+n^{2}-\left(s^{2}+s(a-b) m+1\right) \\
& =\left(a^{2}+1\right) m^{2}+(a+b) s m+1 ;
\end{aligned}
$$

altogether these orbits form a set of dimension

$$
p(a m, m)+o(m, n)=(a+b)(a m+s) m,
$$

and this is just the dimension of $\mathscr{R}(m, n)$. Thus, the set of representations of the form $V \oplus \oplus_{S} S$ with the given properties is dense in $\mathscr{R}(m, n)$, and since these representations have equal endomorphism ring dimension $(n-a m)(n-b m)+1$, we conclude that this has to be $e(n, m)$. A similar calculation gives the value of $e(n, m)$ for $0<a n<m$.

The first two assertions of (c) are immediate consequences of the formula for the endomorphism ring dimension in Proposition 3. Next, let $V$ be indecomposable and in the open sheet. Consider the case of the dimension type $(n, m)$ with $0<m<\frac{1}{a} n$. Now

$$
\begin{aligned}
& \operatorname{dim}_{k} \operatorname{Hom}(S, V) \geqslant\langle S, V\rangle=n-a m, \\
& \operatorname{dim}_{k} \operatorname{Hom}(V, S) \geqslant\langle V, S\rangle=n-b m .
\end{aligned}
$$

As before, let $V^{-S}$ be the intersection of all maps $V \rightarrow S$, and $V^{\prime}$ the sum of all images of maps $S \rightarrow V$. Since $V$ does not split off a copy of $S$, we see that $V^{\prime} \subseteq V^{-S}$, and the ideal $I$ in End $(V)$ of all endomorphisms of $V$ which factor through some $\oplus S$, has dimension

$$
\operatorname{dim}_{k} \operatorname{Hom}(V, S) \cdot \operatorname{dim}_{k} \operatorname{Hom}(S, V) \geqslant(n-a m)(n-b m) .
$$

By assumption,

$$
\operatorname{dim}_{k} \operatorname{End}(V)=(n-a m)(n-b m)+1 ;
$$

thus $I$ has to be the radical of End $(V)$, and in $\left({ }^{* *}\right)$, we must have equality. Since $n>a m \geqslant b m$, we see that also in $\left(^{*}\right)$, both are equalities, and therefore $\operatorname{Ext}(S, V)=0=\operatorname{Ext}(V, S)$. It follows that $V$ belongs to $\mathfrak{M}_{S}^{S}$ and that $\sigma_{S}(V)$ is a representation with trivial endomorphism ring, using Proposition 3. Thus, according to our theorem, either $\sigma_{1}(n, m)=(0,1)$ and then $(n, m)=(a+b, 1)$, or

$$
\sigma_{1}(n, m) \in\left\{(x, y) \mid \frac{1}{a} x \leqslant y \leqslant a x\right\}, \text { thus } \frac{a}{a^{2}+a b-1} n \leqslant m \leqslant \frac{1}{b} n .
$$

Similarly, for $0<a n<m$, we conclude that $V \in \mathfrak{M}_{T}^{T}$, with $\sigma_{T}(V)$ having a trivial endomorphism ring, and then either $(n, m)=(1, a+b)$, or $b n \leqslant m \leqslant \frac{a^{2}+a b-1}{a} n$.

Finally, let us remark that for $X \in \mathfrak{M}_{-S}^{-S}$, the change of endomorphism rings from End $(X)$ to End $\sigma_{S}(X)$ usually increases the nilpotence class of the radical. For example, it is easy to see that $\left(\operatorname{rad} \operatorname{End} \sigma_{S} \sigma_{T}(S)\right)^{2} \neq 0$, whereas $\left(\operatorname{rad} \operatorname{End} \sigma_{T}(S)\right)^{2}=0$. 


\section{Further applications of the reflection functors}

Let us remark that we may use the reflection functors also in the case of a finite dimensional hereditary algebra. Of course, in this case we will also use non-simple modules $S$.

Recall that in the definition of $\mathcal{M l}^{S}$ we had to require as well as $\operatorname{Ext}(S, X)=0$ the additional condition that $X$ cannot be embedded into some $\oplus S$. For example, let $S$ be the unique indecomposable representation of the quiver

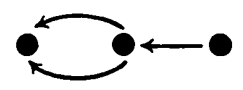

with dimension type (120). The $S$-reflection is defined for a large class of representations, for example for the generic representations of dimension type $(x y=)$ with $3 x<2 y, 2 z<x$. However, the indecomposable representation $X$ of dimension type (230) satisfies on the one hand $\operatorname{Ext}(S, X)=0$, but does not belong to $\mathcal{M} \mathcal{N}^{S}$, since $X$ can be embedded into $S \oplus S$.

Let us give two sets of conditions which imply that a module $X$ with $\operatorname{Ext}(S, X)=0$ belongs to $\mathfrak{M P}^{S}$.

LeMma 7. Let $S$ be a module with endomorphism ring a division ring. If $X$ is an indecomposable module with $\operatorname{Hom}(S, X) \neq 0$, then $X$ cannot be embedded into a direct sum of copies of $S$, or else $X \approx S$.

Proof. Assume that there is an embedding $\mu: X \rightarrow \bigoplus_{i=1}^{n} S$, and let $\varphi: S \rightarrow X$ be non-zero. Then for one of the projections $\pi_{i}$, we see that $\varphi \mu \pi_{i}$ is an automorphism of $S$, so that $\varphi$ is a split monomorphism.

Call a module $X$ S-clean provided that it satisfies the following two conditions:

(i) Precisely one of $\operatorname{Hom}(S, X)$ and $\operatorname{Ext}(X, S)$ is non-zero.

(ii) Precisely one of $\operatorname{Hom}(X, S)$ and $\operatorname{Ext}(S, X)$ is non-zero.

If $S$ is a module with endomorphism ring a division ring and $\operatorname{Ext}(S, S)=0$, then it follows that for all $S$-clean modules the $S$-reflection is defined.

Proposition 5. Let $S$ be a module with End $(S)$ a division ring and $\operatorname{Ext}(S, S)=0$. Let $T$ be any module with $\langle S, T\rangle \leqslant 0,\langle T, S\rangle \leqslant 0$. Then for any indecomposable module $X$ with $\operatorname{dim} X$ a linear combination of $\operatorname{dim} S$ and $\operatorname{dim} T$, and $\operatorname{Ext}(S, X)=0$, we have either $X \approx S$, or else $X$ is not embeddable into a direct sum of copies of $S$.

Proof. Assume that $X$ is embeddable into a direct sum of copies of $S$, and not isomorphic to $S$. Then $\operatorname{Hom}(S, X)=0$, since otherwise $X$ would split off a copy of $S$. Let $\mu_{1}, \ldots, \mu_{v}$ be a basis of $\operatorname{Hom}(X, S)_{\text {End(S) }}$, and $\mu=\left(\mu_{1}, \ldots, \mu_{v}\right)$. Denote by $Y$ the cokernel of $\mu$ : thus we have the exact sequence

$$
0 \longrightarrow X \stackrel{\mu}{\longrightarrow} \bigoplus_{v} S \stackrel{\pi}{\longrightarrow} Y \longrightarrow 0 .
$$


If $\left(\alpha_{i}\right): \bigoplus_{v} S \rightarrow S$ has $\mu(X)$ in its kernel, then $\sum_{i=1}^{v} \mu_{i} \alpha_{i}=0$ implies that $\alpha_{1}=\ldots=\alpha_{v}=0$, since the $\mu_{i}$ are linearly independent. Thus $\operatorname{Hom}(Y, S)=0$.

Also, $\operatorname{Ext}(Y, S)=0$. For the exact sequence above induces an exact sequence

$$
\operatorname{Hom}\left(\bigoplus_{v} S, S\right) \stackrel{\operatorname{Hom}(\mu, S)}{\longrightarrow} \operatorname{Hom}(X, S) \longrightarrow \operatorname{Ext}(Y, S) \longrightarrow \operatorname{Ext}\left(\bigoplus_{v} S, S\right)
$$

the last group being zero by assumption. Also any $\varphi \in \operatorname{Hom}(X, S)$ is a linear combination $\varphi=\sum_{i=1}^{v} \mu_{i} \alpha_{i}$ with $\alpha_{i} \in \operatorname{End}(S)$, thus it is the image of

$$
\left(\begin{array}{c}
\alpha_{1} \\
\vdots \\
\alpha_{v}
\end{array}\right) \in \operatorname{Hom}\left(\bigoplus_{v} S, S\right)
$$

under $\operatorname{Hom}(\mu, S)$. Since we see that $\operatorname{Hom}(\mu, S)$ is surjective, it follows that $\operatorname{Ext}(Y, S)=0$.

As a consequence, we have $\langle S, X\rangle=0$ and $\langle Y, S\rangle=0$. Now, let $\operatorname{dim} X=a \operatorname{dim} S+b \operatorname{dim} T$, where at least one of $a, b$ has to be positive. Now

$$
0=\langle S, X\rangle=a\langle S, S\rangle+b\langle S, T\rangle,
$$

and this can be acheived only if $a, b$ both have the same sign, since $\langle S, S\rangle>0,\langle S, T\rangle \leqslant 0$. Thus, $a \geqslant 0, b>0$. The exact sequence above gives

and

$$
\operatorname{dim} Y=(v-a) \operatorname{dim} S-b \operatorname{dim} T,
$$

$$
0=\langle Y, S\rangle=(v-a)\langle S, S\rangle-b\langle T, S\rangle
$$

implies that $v-a \leqslant 0$, since $\langle S, S\rangle>0, b>0$, and $\langle T, S\rangle \leqslant 0$. But this gives a contradiction since it would mean that $\operatorname{dim} Y$ is a vector with negative components.

\section{References}

1. I. N. Bernstein, I. M. Gelfand and V. A. Ponomarev, "Coxeter functors and Gabriel's theorem", Uspechi Mat. Nauk, 28 (1973), 19-33. Translation: Russian Math. Surveys, 28 (1973), 17-32.

2. V. Kac, "Infinite root systems, representations of graphs and invariant theory", to appear.

3. C. M. Ringel, "Representations of $K$-species and bimodules", J. Algebra, 41 (1976), 269-302.

Fakultät für Mathematik,

Universität,

D-48 Bielefeld,

West Germany. 